\title{
Existence of solution and algorithms for a class of bilevel variational inequalities with hierarchical nesting structure
}

\author{
Gaoxi Li' , Zhongping Wan ${ }^{1,2^{*}}$, Jia-wei Chen ${ }^{3}$ and Xiaoke Zhao'
}

\section{"Correspondence:}

mathwanzhp@whu.edu.cn

${ }^{1}$ School of Mathematics and

Statistics, Wuhan University, Wuhan,

430072, China

${ }^{2}$ Computational Science Hubei Key

Laboratory, Wuhan University, Wuhan, 430072, China

Full list of author information is available at the end of the article

\section{然 Springer}

\begin{abstract}
In this paper we consider a class of bilevel variational inequalities with hierarchical nesting structure. We first of all get the existence of a solution for this problem by using the Himmelberg fixed point theorem. Then the uniqueness of the solution for an upper-level variational inequality is given under some mild conditions. By using gap functions of the upper-level and lower-level variational inequalities, we transform bilevel variational inequalities into a one-level variational inequality. Moreover, we propose two iterative algorithms to find the solutions of the bilevel variational inequalities. Finally, the convergence of the proposed algorithm is derived under some mild conditions.
\end{abstract}

MSC: $90 \mathrm{C} 33 ; 49 J 40 ; 90 C 30$

Keywords: bilevel variational inequalities; Himmelberg fixed point theorem; existence; gap function; iterative algorithm; convergence

\section{Introduction}

The idea of bilevel programming problem may be considered to date back to 1934 when it had been formulated by Stackelberg in a monograph on market economy [1,2]. Since it was introduced to the optimization community in the 1970s, a rapid development and intensive investigation of these problems begun in theoretical analysis [3-9]. Now it has been applied to industry [10], decision science [11], transportation [12], network [13], electricity [14], support chain management [15, 16], cloud computing market [17], and so on. Some mathematical programming problems can be transformed into variational inequality problems. In order to study the bilevel models better, it is necessary to study bilevel variational inequalities.

Bilevel variational inequalities models have been investigated in recent decades (see e.g. [18-24] and references therein). Many algorithms had been constructed to get the approximate solution of these models. These models can well be applied. An imperfection in these models is that the upper-level variational inequality and lower-level variational inequality of all these models are not better embedded in each other. Sometimes the two levels of a bilevel problem are interactional; therefore, the bilevel variational inequalities models, of which the upper -level's variable is embedded into the lower level, and also the lower level's variable is embedded into the upper level, need to be studied.

(c) $2016 \mathrm{Li}$ et al. This article is distributed under the terms of the Creative Commons Attribution 4.0 International License (http://creativecommons.org/licenses/by/4.0/), which permits unrestricted use, distribution, and reproduction in any medium, provided you give appropriate credit to the original author(s) and the source, provide a link to the Creative Commons license, and indicate if changes were made. 
In [25], Wan and Chen introduced bilevel variational inequalities (shortly BVIs) which has a hierarchical nesting structure. They gave the existence theorem of a solution and constructed an algorithm, in the case that the solution set of the lower-level variational inequality $M(x)$ is a singleton $(M(x)$ is the solution set of a lower-level variational inequality, in regard to each parameter $x$ from the upper-level variational inequality). But sometimes, $M(x)$ is not a singleton. In this case, one wonders whether the solution of the BVI exists. How to construct an algorithm? In this paper, our work will consider the two problems.

Now we review the model in [25]. Let $K$ be nonempty subset of the $n$-dimensional Euclidean space $R^{n}$, and $H: R^{m} \times R^{n} \rightarrow R^{n}$ and $P: R^{m} \rightarrow R^{m}$ be two mappings. Let $T: K \rightarrow 2^{R^{m}}$ be a set-valued mapping, where $2^{R^{m}}$ is the family of all nonempty subsets of $R^{m}$.

Consider the following bilevel variational inequalities (shortly BVIs): find $(x, z) \in K \times R^{m}$, such that

$$
\langle H(z, x), x-y\rangle \leq 0, \quad \forall y \in K
$$

where $z \in M(x), M(x)$ is the solution set of the following parametric variational inequality: find $z \in T(x)$ such that

$$
\langle P(z), z-v\rangle \leq 0, \quad \forall v \in T(x)
$$

Inequations (1) and (2) are called the upper-level variational inequality (shortly (UVI)) and the lower-level variation inequality (shortly (LVI)), respectively. The decision variables of the problem BVI are divided into two classes, namely, the upper-level decision variable $x$ and the lower-level decision variable $z$. Denote the optimal solution set of the BVI by $\Theta$.

Obviously, the BVI involves two variational inequalities. The constraint region of the lower-level variational inequality $T(x)$ is implicitly determined by the parameter $x$ from upper-level variational inequality. The lower-level decision variable $z$ is embedded into upper-level inequality. It is extremely difficult to solve globally, because of its nested structure. In a broad sense, it is similar to a quasi-variational inequality [26-28].

Next we review some definitions that are referred in [25].

The solution set of the lower-level variational inequality in regard to the parameter $x$ from the upper-level variational inequality is

$$
M(x)=\{z \in T(x):\langle P(z), z-v\rangle \leq 0, \forall v \in T(x)\} .
$$

The solution set of the upper-level variation inequality for every parameter $z$ from the lower-level variational inequality is

$$
U(z)=\{x \in K:\langle H(z, x), x-y\rangle \leq 0, \forall y \in K\} .
$$

We call the BVI well-posed bilevel variational inequalities, if $M(x)$ is a singleton set. We call it ill-posed bilevel variational inequalities, if $M(x)$ has more than one element. Wan and Chen gave the existence of the solution and an algorithm when the set $M(x)$ is a singleton in [25]. But sometimes, $M(x)$ is not a singleton set; this can be seen from the next example. 
Example 1.1 Let $R=(-\infty,+\infty), K=[-2,-1]$ and $T(x)=[-2, x]$ for all $x \in K, z, v, x, y \in$ $R$. Let $\langle P(z), z-v\rangle=\left\langle-(z+1)(2 z+3)(3 z+4) e^{z}, z-v\right\rangle=-(z+1)(2 z+3)(3 z+4) e^{z}(z-v)$, $\langle H(z, x), x-y\rangle=\left\langle-(z+2)(z+1) x e^{z}, x-y\right\rangle=-(z+2)(z+1) x e^{z}(x-y)$. The BVI is defined as follows: find $x \in K$ such that

$$
-(z+2)(z+1) x e^{z}(x-y) \leq 0, \quad \forall y \in K,
$$

where $z$ is a solution of the following variational inequality: find $z \in T(x)$ such that

$$
-(z+1)(2 z+3)(3 z+4) e^{z}(z-v) \leq 0, \quad \forall v \in T(x) .
$$

A simple computation shows that the solution set of lower-level variational inequality is

$$
M(x)= \begin{cases}\{-2\}, & \text { if } x \in\left[-2,-\frac{3}{2}\right), \\ \left\{-\frac{3}{2}\right\}, & \text { if } x \in\left[-\frac{3}{2},-\frac{4}{3}\right), \\ \left\{-\frac{3}{2},-\frac{4}{3}\right\}, & \text { if } x \in\left[-\frac{4}{3},-1\right), \\ \left\{-2,-\frac{3}{2},-\frac{4}{3}\right\}, & \text { if } x=-1 .\end{cases}
$$

We can see that if $x \in\left[-\frac{4}{3},-1\right)$, then $M(x)=\left\{-\frac{3}{2},-\frac{4}{3}\right\}$; there are two elements in $M(x)$. So it is not a singleton set. It is easy to verify that $\left\{(-2, x): x \in\left[-2,-\frac{3}{2}\right)\right.$ or $\left.x=-1\right\}$ is the solution set of the BVI (3)-(4). So it is meaningful to research the existence theorem of the solution and algorithm when the lower-level solution set $M(x)$ is not a singleton. This paper will discuss these questions.

The rest of this paper is organized as follows. In Section 2, we recall some important results of [25] and give some preliminary definitions which are needed for our main results. In Section 3, we demonstrate the existence theorem of a solution when the lowerlevel solution set $M(x)$ is not a singleton, and we investigate the unique solution condition of the upper-level variational inequality for every parameter $z$ from the lower-level variational inequality. In Section 4, we transform the BVI into a one-level variational inequality by gap functions of the lower-level variational inequality and the upper-level variational inequality. Based on Section 4, we proposed two iterative algorithms to compute the approximation solution of the BVI, and analyzed the convergence of the presented algorithm in Section 5. Some numerical examples are given in Section 6.

\section{Preliminaries}

We first review some definitions and lemmas which are needed for our main results.

Definition 2.1 ([25]) Let $\Xi$ be a nonempty subset of $R^{m}$. A mapping $P: \Xi \rightarrow R^{m}$ is said to be

(i) monotone on $\Xi$ if

$$
\langle P(x)-P(y), y-x\rangle \leq 0, \quad \forall(x, y) \in \Xi \times \Xi ;
$$

(ii) strictly monotone on $\Xi$ if

$$
\langle P(x)-P(y), y-x\rangle<0, \quad \forall(x, y) \in \Xi \times \Xi, x \neq y ;
$$


(iii) pseudomonotone if for any $(x, y) \in \Xi \times \Xi$

$$
\langle P(x), y-x\rangle \geq 0 \Rightarrow\langle P(y), x-y\rangle \leq 0 .
$$

It is easy to see that

strict monotonicity $\Rightarrow$ monotonicity $\Rightarrow$ pseudomonotonicity.

Definition 2.2 Let $\Lambda$ be a nonempty subset of $R^{n}$. Let $\Xi$ be a nonempty subset of $R^{m}$, for all $z \in \Lambda$, a function $H: \Lambda \times R^{n} \rightarrow R^{n}$ is said to be

(i) monotone on $\Xi$ if

$$
\langle H(z, x)-H(z, y), y-x\rangle \leq 0, \quad \forall(x, y) \in \Xi \times \Xi
$$

(ii) strictly monotone on $\Xi$ if

$$
\langle H(z, x)-H(z, y), y-x\rangle<0, \quad \forall(x, y) \in \Xi \times \Xi, x \neq y
$$

Definition 2.3 ([29]) Let $X \subseteq R^{n}, Y \subseteq R^{m}$. A set-valued mapping $T: X \rightarrow 2^{Y}$ is said to be

(i) upper semicontinuous (shortly, usc) at $x_{0} \in X$ if, for each open set $V$ with $T\left(x_{0}\right) \subset V$, there exists $\delta>0$ such that

$$
T(x) \subset V, \quad \forall x \in B\left(x_{0}, \delta\right)
$$

(ii) lower semicontinuous (shortly, lsc) at $x_{0} \in X$ if, for each open set $V$ with $T\left(x_{0}\right) \cap V \neq \emptyset$, there exists $\delta>0$ such that

$$
T(x) \cap V \neq \emptyset, \quad \forall x \in B\left(x_{0}, \delta\right)
$$

(iii) closed if the graph of $T$ is closed, i.e., the set $\operatorname{Gr}(T)=\{(\zeta, x) \in P \times E: \zeta \in T(x)\}$ is closed in $X \times Y$.

We say $T$ is lsc (resp. usc) on $X$ if it is lsc (resp. usc) at each $x \in X . T$ is called continuous at $X$ if it is both lsc and usc on $X$.

Definition 2.4 ([29]) Let $K$ be a nonempty convex subset of $R^{m}$. A set-valued mapping $T: K \rightarrow 2^{R^{m}}$ is said to be convex-valued (compact-valued, closed-valued) if, the images $T(x)$ of all points $x \in K$ are convex (compact, closed).

Definition 2.5 ([30]) Let $\Omega \in R^{n}, E \in R^{m}$. A set-valued mapping $G: \Omega \rightarrow 2^{E}$ is said to be local intersection if, for any $x \in \Omega$ there exists an open neighborhood $U_{x}$ of $x$, such that $\bigcap_{x^{\prime} \in U_{x}} G\left(x^{\prime}\right) \neq \emptyset$. We denote $G(\Omega)=\bigcup_{x \in \Omega} G(x)$.

Lemma 2.1 (Continuous selection theorem [30,31]) Let $X \subseteq R^{n}, Y$ be a nonempty paracompact subset of $R^{m}$. Let $G, M: X \rightarrow 2^{Y}$ be two set-valued mappings. Assume that the following conditions hold:

(i) For any $x \in X, G(x)$ is nonempty, and $\operatorname{co}(G(x)) \subset M(x)$;

(ii) $G$ is local intersection. 
Then $M$ has a continuous selection, namely, there is a continuous mapping $f: X \rightarrow Y$, such that $f(x) \in M(x), \forall x \in X$.

Remark 2.1 ([31]) If $X$ is a compact set, then $c o X$ is a paracompact set.

Lemma 2.2 ([29]) Let $X \subseteq R^{n}, Y \subseteq R^{m}, F, G: X \rightarrow 2^{Y}$ be two set-valued mappings such that, for all $x \in X, F(x) \cap G(x) \neq \emptyset$. We suppose that:

(i) $F$ is upper semicontinuous at $x_{0}$;

(ii) $F\left(x_{0}\right)$ is compact;

(iii) $G$ is closed.

Then the set-valued map $F \cap G: x \rightarrow F(x) \cap G(x)$ is upper semicontinuous at $x_{0}$.

Lemma 2.3 (Himmelberg fixed point theorem [32,33]) Let $X$ be a convex subset of $R^{m}$. $D$ is a nonempty compact subset of $X, H: X \rightarrow 2^{D}$ is an upper semicontinuous set-valued mapping. And for all $x \in X, H(x)$ is a nonempty closed convex subset of $D$. Then there is a point $\bar{x} \in D$, such that $\bar{x} \in H(\bar{x})$.

Lemma 2.4 ([34]) Let $K$ be a nonempty subset of $R^{m}$. A set-valued mapping $T: K \rightarrow 2^{R^{m}}$ is compact-valued and continuous. $f: K \times R^{n}$ is continuous. Then

$$
N(x):=\{z \in T(x): \forall y \in T(x), f(x, z) \geq f(x, y)\}
$$

defines a compact-valued, and $n(x):=\max _{y \in T(x)} f(x, y)$ is continuous.

\section{Existence of solution for the BVI}

In this section, we investigate the existence of solution for the BVI when the lower-level solution set $M(x)$ is not a singleton under some suitable conditions.

For simplicity, let $\phi: R^{m} \times R^{n} \times R^{n} \rightarrow R^{n}, \phi: R^{m} \times R^{n} \times R^{n} \rightarrow R^{n}$, we denote the following equations:

$$
\begin{aligned}
& \phi(z, x, y)=\langle H(z, x), x-y\rangle, \\
& \psi(z, v)=\langle P(z), z-v\rangle .
\end{aligned}
$$

Remark 3.1 It is easy to verify that $\phi(z, x, x)=\langle H(z, x), x-x\rangle=0$, both $v \mapsto \psi(\cdot, v)$ and $y \mapsto$ $\phi(\cdot, \cdot, y)$ are concave and continuous. For each $w, v \in R^{m}, z \mapsto\langle P(w), z-v\rangle$ is continuous.

Lemma 3.1 ([25]) Let $K$ be nonempty convex subset of $R^{n}, T: K \rightarrow 2^{R^{m}}$ be closed convexvalued. Assume that the following conditions hold:

(i) $P$ is monotone;

(ii) $z \mapsto \psi(z, \cdot)$ is lower-hemicontinuous.

Then for each $x \in K$, the solution set $M(x)$ of $(L V I)$ is closed and convex.

Since it is easy to verify that $\psi$ and $P$ in Example 3.2 satisfies all conditions of Lemma 3.1, and $M(x)$ is a closed and convex set, from Example 3.2 we can see that Lemma 3.1 is applicable.

Lemma 3.2 ([25]) Let $K$ be nonempty convex subset of $R^{n}, T: K \rightarrow 2^{R^{m}}$ be convex and compact-valued. Assume that the following conditions hold: 
(i) P is pseudomonotone;

(ii) $z \mapsto \psi(z, \cdot)$ is lower-hemicontinuous.

Then for each $x \in K$, the solution set $M(x)$ of $(L V I)$ is nonempty and compact.

Example 3.1 can be used to show that the result of Lemma 3.2 is also applicable.

Example 3.1 Let $R=(-\infty,+\infty), K=\left[-1,-\frac{1}{2}\right], T(x)=[x, 1]$ for $x \in K$, and let $\langle P(z), z-v\rangle=$ $\left\langle e^{z}, z-v\right\rangle$. It is obvious that all conditions of Lemma 3.2 are satisfied. By computation, for each $x \in K$, the lower-level solution set $M(x)=\{x\} . M(x)$ is a nonempty and compact set.

Remark 3.2 Since monotonicity $\Rightarrow$ pseudomonotonicity, from the conditions of Lemma 3.2, we can further see that, for each $x \in K$, the solution set $M(x)$ of (LVI) is convex nonempty and compact.

Remark 3.3 Under the conditions of the Lemma 3.2, $M(x)$ may be not a singleton set, this can be seen in Example 3.2. This example also shows that Lemma 3.2 is applicable.

Example 3.2 Let $R=(-\infty,+\infty), K=[0.5,6] \times[0.5,6], x=\left(x_{1}, x_{2}\right)^{\top}, x=\left(y_{1}, y_{2}\right)^{\top}, z=$ $\left(z_{1}, z_{2}\right)^{\top}, v=\left(v_{1}, v_{2}\right)^{\top}$, and $T(x)=\left\{y: 0<y_{1}<1+x_{1}, 0<y_{2}<1+x_{2}, y_{1}+y_{2}=1\right\}$ for $x \in K$, $z, v \in R^{2}$. Let $P(z)^{\top}=(1,1)$.

Obviously, $P$ satisfies the conditions of Lemma 3.2. By a simple computation one gets the solution set of (LVI),

$$
M(x)=\left\{z: 0 \leq z_{1}, 0 \leq z_{2}, z_{1}+z_{2}=1\right\}
$$

It is obvious that $M(x)$ is nonempty convex compact and not a singleton.

The next theorem shows the existence of solution when the solution set $M(x)$ of (LVI) is not a singleton. That is, the BVI is an ill-posed bilevel variational inequality.

Theorem 3.1 Suppose that $K$ is a nonempty compact convex subset of $R^{m} ; T: K \rightarrow 2^{R^{m}}$ is a convex and compact-valued set-valued mapping, and, for all $x \in K, K \in T(x)$. Assume that the following conditions hold:

(i) $P$ is pseudomonotone;

(ii) $z \mapsto \psi(z, \cdot)$ is lower-hemicontinuous;

(iii) $\inf _{z \in K} \sup _{v \in T(K)} \psi(z, v) \leq 0$;

(iv) the function $\phi$ is a continuous function.

Then there exist $\bar{x} \in K, \bar{z} \in M(\bar{x})$, such that $\langle H(\bar{z}, \bar{x}), \bar{x}-y\rangle \leq 0, \forall y \in K$. That is, the BVI (1)-(2) has at least one solution $(\bar{z}, \bar{x})$.

Proof By conditions (i), (ii), and Lemma 3.2, it is easy to show that the solution set $M(x)$ of (LVI) is nonempty, convex, and compact. Since $K$ is a nonempty convex compact set, from condition (ii) and (iii) we see that there exists $z_{0} \in K$ satisfying

$$
\sup _{v \in T(K)} \psi\left(z_{0}, v\right)=\inf _{z \in K} \sup _{v \in T(K)} \psi(z, v) \leq 0
$$


First, we will show that $z_{0} \in \bigcap_{x \in K} M(x)$. Suppose by contradiction that $z_{0} \notin \bigcap_{x \in K} M(x)$, then there exists $x^{\prime} \in K$ such that $z_{0} \notin M\left(x^{\prime}\right)$. That is,

$$
z_{0} \notin M\left(x^{\prime}\right)=\left\{z \in T\left(x^{\prime}\right): \psi(z, v) \leq 0, \forall v \in T\left(x^{\prime}\right)\right\}
$$

so there exists at least one $v^{\prime} \in T\left(x^{\prime}\right)$, such that $\psi\left(z_{0}, v^{\prime}\right)>0$. In view of condition (iii), we get

$$
\psi\left(z_{0}, v^{\prime}\right) \leq \sup _{v \in T(K)} \psi\left(z_{0}, v\right)=\inf _{z \in K} \sup _{v \in T(K)} \psi(z, v) \leq 0,
$$

which is a contradiction.

Second, we show that set-valued mapping $M$ satisfies the conditions of Lemma 2.1 (continuous selection theorem). In fact, since, for any $x \in K, M(x)$ is nonempty convex compact set, it is obvious that there exists a nonempty convex compact subset $N_{x}$ for $x$, satisfying $z_{0} \in N_{x} \subset M(x)$, then we can find a set-valued mapping $G: K \mapsto 2^{R^{m}}$, for every $x \in K$, such that $G(x)=N_{x}$. Obviously, $G$ satisfies Definition 2.5. It easy to see that the following two conditions are satisfied:

(i) For any $x \in K, G(x)$ is nonempty, and $c o(G(x))=c o\left(N_{x}\right) \subset M(x)$;

(ii) $\bigcap_{x \in K} G(x)=\bigcap_{x \in K} N_{x} \neq \emptyset$,

that is, $M$ satisfies Lemma 2.1. Then, from Lemma 2.1 and Remark 2.1 we know that there is a continuous mapping $f: K \mapsto R^{m}$ such that $f(x) \in M(x), \forall x \in K$.

For every $x \in K$, suppose

$$
F(x)=\left\{\hat{y} \in K: \phi(f(x), x, \hat{y})=\max _{y \in K} \phi(f(x), x, y)\right\}
$$

Clearly, $F: K \rightarrow 2^{K}$ is a set-valued mapping. According to the continuous property of $f$ and $\phi$, it follows that $y \rightarrow \phi(f(x), x, y)$ is continuous. By the compactness property of $K$, we know that there exists at least one $\hat{y} \in K$ such that

$$
\phi(f(x), x, \hat{y})=\max _{y \in K} \phi(f(x), x, y)
$$

so, $F(x)$ is a nonempty set.

Next, we will show that the set-valued mapping $F: K \rightarrow 2^{K}$ is convex-valued, compactvalued, and upper-semicontinuous.

First of all, we show that $F$ is convex-valued. For every $x \in K$, suppose $y_{1}, y_{2} \in F(x)$, that is,

$$
\phi\left(f(x), x, y_{1}\right)=\phi\left(f(x), x, y_{2}\right)=\max _{y \in K} \phi(f(x), x, y) .
$$

Let $y_{0}=\lambda y_{1}+(1-\lambda) y_{2}, \forall \lambda \in(0,1)$, Since $y \mapsto \phi(\cdot, \cdot, y)$ is concave, we have

$$
\begin{aligned}
\max _{y \in K} \phi(f(x), x, y) & =\lambda \phi\left(f(x), x, y_{1}\right)+(1-\lambda) \phi\left(f(x), x, y_{2}\right) \\
& \leq \phi\left(f(x), x, y_{0}\right) \leq \max _{y \in K} \phi(f(x), x, y),
\end{aligned}
$$


so

$$
\phi\left(f(x), x, y_{0}\right)=\max _{y \in K} \phi(f(x), x, y), \quad y_{0} \in F(x)
$$

that is, $F(x)$ is a convex set.

Second, we prove that $F$ is compact-valued. For every $(x, y) \in K \times K$, let $\eta(x, y)=$ $-\phi(f(x), x, y)$. It is easy to see that $y \rightarrow \eta(x, y)$ is continuous, and

$$
F(x)=\left\{\hat{y} \in K: \eta(x, \hat{y})=\min _{y \in K} \eta(x, y)\right\} .
$$

For every sequence $\left\{y_{k}\right\} \subset F(x), y_{k} \rightarrow y_{0}$, we can get the following equation:

$$
\min _{y \in K} \eta(x, y)=\lim _{k \rightarrow+\infty} \min _{y \in K} \eta(x, y)=\lim _{k \rightarrow+\infty} \eta\left(x, y_{k}\right)=\eta\left(x, \lim _{k \rightarrow+\infty} y_{k}\right)=\eta\left(x, y_{0}\right)
$$

therefore, $y_{0} \in F(x), F$ is compact-valued.

Finally, we show that $F$ is upper semicontinuous. Let $Q: K \rightarrow 2^{K}$ be set-valued mapping satisfying $Q(x)=K, \forall x \in K$. It is obvious that $Q$ is a continuous and compact-valued mapping.

We denote

$$
F(x)=Q(x) \cap J(x)
$$

where $J(x)=\{y: \eta(x, \hat{y})=\eta(x, y)\}$. Due to $J(x)$ is closed. According to Lemma 2.2 we know that $H$ is upper semicontinuous.

From the above discussion, it is obvious to know that $F$ satisfies the conditions of Lemma 2.3 (Himmelberg fixed point theorem), so there exists a point $\bar{x} \in K$, such that $\bar{x} \in F(\bar{x})$. Then the point $\bar{x} \in K$ satisfies

$$
\phi(f(\bar{x}), \bar{x}, \bar{x})=\max _{y \in K} \phi(f(\bar{x}), \bar{x}, y) .
$$

Let $\bar{z}=f(\bar{x})$, then $\bar{z} \in M(\bar{x})$, and $\phi(\bar{z}, \bar{x}, \bar{x})=\max _{y \in K} \phi(\bar{z}, \bar{x}, y)$. So, for every $y \in K$

$$
\phi(\bar{z}, \bar{x}, y) \leq \max _{y \in K} \phi(\bar{z}, \bar{x}, y)=\phi(\bar{z}, \bar{x}, \bar{x})=0
$$

that is, $(\bar{z}, \bar{x}) \in \Theta$, here $\Theta$ is the solution set of the BVI (1)-(2).

Example 3.3 Let $R=(-\infty,+\infty), K=[0.5,6] \times[0.5,6], x=\left(x_{1}, x_{2}\right)^{\top}, z=\left(z_{1}, z_{2}\right)^{\top}, v=$ $\left(v_{1}, v_{2}\right)^{\top}, y=\left(y_{1}, y_{2}\right)^{\top}, u=\left(u_{1}, u_{2}\right)^{\top}$, and $T(x)=\left\{u: 0<u_{1}<1+x_{1}, 0<u_{2}<1+x_{2}, u_{1}+u_{2}=1\right\}$ for $x \in K, z, v, y \in R^{2}$. Let $H(z, x)^{\top}=(1,1) z\left(\frac{1}{2}, \frac{1}{2}\right), P(z)^{\top}=(1,1)$.

It is not hard to see that all conditions of Theorem 3.1 are satisfied.

Simple computation we get the solution set of the lower-level problem

$$
M(x)=\left\{z: 0 \leq z_{1}, 0 \leq z_{2}, z_{1}+z_{2}=1\right\} .
$$

It is obvious that $M(x)$ is not a singleton set. Further, we can see that $M(x)$ is a nonempty convex and compact set, and $\left\{(z, x) \in R_{+}^{2} \times R_{+}^{2}: z_{1}+z_{2}=1, x_{1}+x_{2}=1\right\}$ is the solution set of the BVI. 
Remark 3.4 From Example 3.3 we can verify that $M$ satisfies Lemma 2.1. We can choose the continuous function $f(x)=z, z \in M(x)$. It is obvious that $f(x)$ is a constant function.

We can find the following phenomenon from Example 3.3.

If the upper-level decision maker feedback parameter $x=(0.5,0.5)$ to the lower-level decision maker, then we can get the lower-level solution set $M((0.5,0.5))=\left\{z: 0 \leq z_{1}, 0 \leq\right.$ $\left.z_{2}, z_{1}+z_{2}=1\right\}$. Here, if the lower-level decision maker feedback parameter $z=(0.5,0.5)$ to the upper-level decision maker, then it is well known that $U((0.5,0.5))=\left\{(z, x) \in R_{+}^{2} \times R_{+}^{2}\right.$ : $\left.z_{1}+z_{2}=1, x_{1}+x_{2}=1\right\}$ is the solution set of the upper-level variational inequality, and $U((0.5,0.5))$ is not a singleton set. If the conditions of Theorem 3.1 are strengthened, we will get the unique solution of the upper-level variational inequality, in regard to every parameter $z$ from the lower-level variational inequality. That is, $U(z)$ is a singleton set.

Theorem 3.2 (unique solution of (UVI)) Under the hypotheses of Theorem 3.1, if, moreover, $H(z, x)$ is strictly monotone, for every $z \in R^{m}$, then, for every $z \in M\left(x_{0}\right)$, the solution set of the upper-level variation inequality

$$
U(z)=\{x \in K:\langle H(z, x), x-y\rangle \leq 0, \forall y \in K\}
$$

is a singleton.

Proof For each $x_{0} \in K$ from Theorem 3.1, we know that $M\left(x_{0}\right) \neq \emptyset$. Using a proof by contradiction, suppose that there exists a $z \in M\left(x_{0}\right)$ such that $U(z)$ is not a singleton. That is, there exist $x_{1}, x_{2} \in K, x_{1} \neq x_{2}$ such that

$$
\left\langle H\left(z, x_{1}\right), x_{1}-y\right\rangle \leq 0, \quad \forall y \in K
$$

and

$$
\left\langle H\left(z, x_{2}\right), x_{2}-y\right\rangle \leq 0, \quad \forall y \in K
$$

moreover, we have

$$
\left\langle H\left(z, x_{1}\right), x_{1}-x_{2}\right\rangle \leq 0
$$

and

$$
\left\langle H\left(z, x_{2}\right), x_{2}-x_{1}\right\rangle \leq 0
$$

Since for every $z \in R^{m}, H(z, x)$ is strictly monotone, from (ii) of Definition 2.2, we have

$$
\left\langle H\left(z, x_{2}\right)-H\left(z, x_{1}\right), x_{1}-x_{2}\right\rangle<0,
$$

that is,

$$
\left\langle H\left(z, x_{2}\right), x_{2}-x_{1}\right\rangle+\left\langle H\left(z, x_{1}\right), x_{1}-x_{2}\right\rangle>0 .
$$


From (5) and (7) we have

$$
\left\langle H\left(z, x_{2}\right), x_{2}-x_{1}\right\rangle>0
$$

which contradicts (6). Therefore, $U(z)$ is a singleton.

\section{Equivalence transformation of the BVI}

In this section, we shall transform the bilevel variational inequalities (1) and (2) into a one-level variational inequality by the gap functions of (LVI) and (UVI).

We now define the functions $\alpha: R^{m} \times R^{n} \rightarrow R, \beta: R^{m} \times R^{n} \rightarrow R, g: R^{m} \times R^{n} \rightarrow R$, by

$$
\begin{aligned}
& \alpha(z, x)=\sup _{v \in T(x)}\langle P(z), z-v\rangle, \quad(z, x) \in R^{m} \times R^{n}, \\
& \beta(z, x)=\sup _{y \in K}\langle H(z, x), x-y\rangle, \quad(z, x) \in R^{m} \times R^{n},
\end{aligned}
$$

and

$$
g(z, x)=\beta(z, x)+\alpha(z, x), \quad(z, x) \in R^{m} \times R^{n} .
$$

Remark 4.1 For each $x \in K, z \in M(x)$ if $\alpha(z, x)=0$. Moreover, we obtain that $\alpha(z, x)=0$ from $\alpha(z, x) \geq 0$ for all $z \in T(x)$. The same holds for $\beta(z, x)$.

We consider the parametric variational inequality defined by the bifunction PVI: find $x \in K, z \in T(x)$ such that

$$
g(z, x) \leq 0
$$

Denote the solution set of the PVI by $S$.

Theorem $4.1(x, z) \in \Theta$ if and only if $(x, z) \in S$.

Proof First, we verify that $(x, z) \in \Theta \Rightarrow(x, z) \in S$. Assume that $(x, z) \in \Theta$, then $x \in K, z \in$ $T(x)$,

$$
\langle P(z), z-v\rangle \leq 0, \quad \forall v \in T(x)
$$

and

$$
\langle H(z, x), x-y\rangle \leq 0, \quad \forall y \in K,
$$

further, we have

$$
\begin{aligned}
& \alpha(z, x)=\sup _{v \in T(x)}\langle P(z), z-v\rangle \leq 0, \\
& \beta(z, x)=\sup _{y \in K}\langle H(z, x), x-y\rangle \leq 0,
\end{aligned}
$$


therefore,

$$
g(z, x)=\beta(z, x)+\alpha(z, x) \leq 0,
$$

this implies that $(z, x)$ is a solution of the PVI, so $(z, x) \in S$.

The next thing to do in the proof is to show that $(x, z) \in S \Rightarrow(x, z) \in \Theta$. Let $(z, x) \in S$, then

$$
g(z, x)=\beta(z, x)+\alpha(z, x) \leq 0,
$$

from Remark (4.1) we have

$$
\begin{aligned}
& \alpha(z, x) \geq 0, \quad \forall z \in T(x), \\
& \beta(z, x) \geq 0, \quad \forall x \in K,
\end{aligned}
$$

from (9), (10), and (11), we have $\alpha(z, x)=0, \beta(z, x)=0$, so $(z, x)$ is the solution of the BVI, that is, $(z, x) \in \Theta$.

Corollary 4.1 If all conditions of Theorem 3.1 are satisfied, then $S$ is nonempty and

$$
(z, x) \in \Theta \quad \Leftrightarrow \quad(z, x) \in S
$$

\section{Algorithm and convergence analysis}

In this section, we first present Algorithm 1 for the BVI (1) and (2) from the theoretical point of view, and then consider the limiting behavior of the sequence generated by the algorithm. Algorithm 1 can be used to solve the BVI which has only one solution. In order to solve the BVI which has more than one solution we modify Algorithm 1 to obtain Algorithm 2.

We have transformed the BVI (1) and (2) into a single-level variational inequality (PVI) (8), and discussed the relationship between the two problems in the previous section. From Theorem 4.1, we know that the two problems are equivalent. So we can find the solution of the BVI (1) and (2) by solving the PVI problem (8). In Algorithm 1, we will solve the BVI (1)-(2) by solving a sequence of the approximation problem PVI (8).

\section{Algorithm 1}

Step 1. Take $\left\{\mu_{k}\right\}_{k \in Z^{+}} \subset(1,+\infty)$, where $Z^{+}$is the set of all nonnegative integers. Choose $x_{0} \in K$ arbitrarily, let $m=x_{0}$, compute $z_{0}=\arg \min _{z \in T\left(x_{0}\right)} g\left(x_{0}, z\right)$. Let the tolerance $\varepsilon \geq 0$, $k=0$. If $g\left(z_{0}, x_{0}\right) \leq \varepsilon$, stop, put out $\left(z_{0}, x_{0}\right)$. Otherwise, go to Step 2 .

Step 2. Let $K=K \backslash m$, take $m \in K$. Compute $n=\arg \min _{z \in T(m)} g(z, m)$, go to Step 3 .

Step 3. If

$$
g(n, m) \leq \frac{1}{\mu_{k}} g\left(z_{k}, x_{k}\right),
$$

let $z_{k+1}=n, x_{k+1}=m$ go to Step 4. Otherwise, go to Step 2 .

Step 4. If

$$
g\left(z_{k+1}, x_{k+1}\right) \leq \varepsilon,
$$

stop, put out $\left(z_{k+1}, x_{k+1}\right)$. Otherwise, let $k=k+1$ go to Step 2 . 
Remark 5.1 In Step 2, we discretize $K$ within acceptable error range of the solution. We take different discrete points $m$ of $K$ in every iterative process. For example, if the error band of solution is $2 \delta, K \in R$, then in every iterative process, $m=m+\delta$ (or $m=m-\delta$ ). If with the algorithm we have not found a solution until $m$ has equality to one of the boundary points of $K$, put the other boundary point in $m$. Algorithm 1 is effective for the bilevel variational inequalities which have only one solution.

Remark 5.2 In order to guarantee the existence of a solution for $\min _{z \in T(m)} g(z, m)$ in Step 2, we need to suppose that $\psi$ and $\phi$ are continuous and $T$ is compact-valued and continuous. Then according to Lemma 2.4, it follows that $z \mapsto \alpha(z, x)$ and $z \mapsto \beta(z, x)$ are continuous; further, $z \mapsto g(z, x)$ is continuous. Thus $\min _{z \in T(m)} g(z, m)$ has a solution.

To explain the Algorithm 1, we give the next example.

Example 5.1 Let $R=(-\infty,+\infty), K=[-2,-1]$, and $T(x)=[-2, x]$ for $x \in K, z, v, y \in R$. $\langle H(z, x), x-y\rangle=\left\langle z x e^{z}, x-y\right\rangle=z x e^{z}(x-y),\langle P(z), z-v\rangle=\left\langle-(2 z+3) e^{z}, z-v\right\rangle=-(2 z+3) e^{z}(z-v)$. The BVI is defined as follows: find $x \in K$ such that

$$
z x e^{z}(x-y) \leq 0, \quad \forall y \in K
$$

where $z$ is the solution of the following variational inequality: find $z \in T(x)=[-2, x]$ such that

$$
-(2 z+3) e^{z}(z-v) \leq 0, \quad \forall v \in T(x)
$$

By a simple computation we get the lower-level solution set,

$$
M(x)= \begin{cases}\{-2\}, & \text { if } x \in\left[-2,-\frac{3}{2}\right), \\ \left\{-2,-\frac{3}{2}\right\}, & \text { if } x \in\left[-\frac{3}{2},-1\right] .\end{cases}
$$

Observe that $\mathrm{M}(\mathrm{x})$ is not a singleton, and $(-2,-2)$ is the unique solution of the BVI (13)-(14). By the proposed Algorithm 1, first we should transform the BVI into a one-level variational inequality.

The gap function of the lower-level variational inequality is

$$
\alpha(z, x)=\sup _{v \in[-2, x]}-(2 z+3) e^{z}(z-v) .
$$

Moreover,

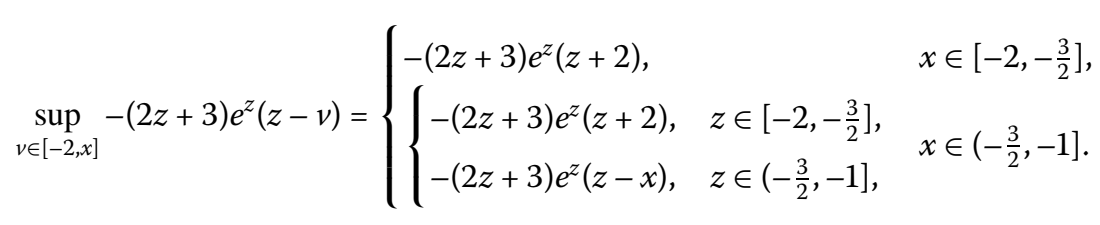

The gap function of upper-level variational inequality is

$$
\beta(z, x)=\sup _{y \in[-2,-1]} z x e^{z}(x-y)=z x e^{z}(x+2) .
$$


By equality (9), we have

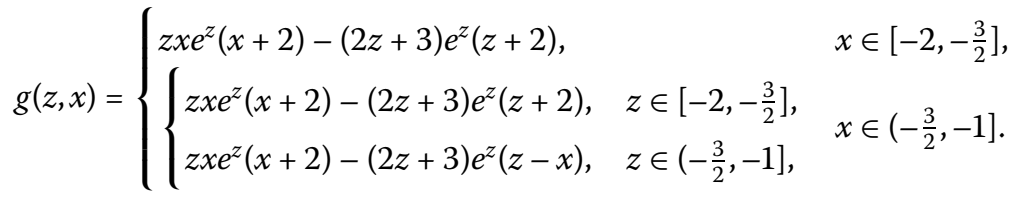

Next, we illustrate the process of solving the BVI (13)-(14) by the proposed Algorithm 1. Step 1 . Choose $x_{0}=-\frac{3}{2}$ and the tolerance $\varepsilon=10^{-5}, \mu_{k}=\mu=2$.

Step 2 . Verify whether there exists $z_{0} \in T\left(x_{0}\right)=\left[-2,-\frac{3}{2}\right]$ such that inequality (12) holds. From equality (15) we have

$$
g\left(z, x_{0}\right)=z x_{0} e^{z}\left(x_{0}+z\right)-(2 z+3) e^{z}(z+2)
$$

In order to find $z_{0} \in T\left(x_{0}\right)$, we need to solve the following optimization problem:

$$
\min _{z \in T\left(x_{0}\right)} g\left(z, x_{0}\right)
$$

Applying the descent direction method to solve (16), we conclude that $z_{0}=-2$, which is an optimal solution of (16), but

$$
g\left(z_{0}, x_{0}\right)=2 x_{0} e^{z_{0}}\left(x_{0}+2\right)-\left(2 z_{0}+3\right) e^{z_{0}}\left(z_{0}+2\right)=\frac{3}{2 e^{2}}>\varepsilon .
$$

Go to Step 2, set $K=K \backslash\left\{-\frac{3}{2}\right\}$. For simplicity, take $m=-2 \in K$. In numerical experiments, we take $\mathrm{m}$ by the method of Remark 5.1. Solve the following optimization problem:

$$
\arg \min _{n \in T(2)} g(n, 2)
$$

Applying the descent direction method to solve (17), we get $n=-2$, and go to Step 3 . Step 3. We have

$$
g(n, m) \leq \frac{1}{2} g\left(z_{0}, x_{0}\right)
$$

Let $z_{1}=n, x_{1}=m$, go to Step 4 .

Step 4. As $g\left(z_{1}, x_{1}\right)=0 \leq \varepsilon$, stop, put out $\left(z_{1}, x_{1}\right)$. So $(-2,-2)$ is the solution of the BVI (13)-(14).

\subsection{Convergence analysis}

Let us consider the behavior of a sequence $\left\{\left(x_{k}, z_{k}\right)\right\}$ generated by the proposed Algorithm 1 .

Theorem 5.1 Suppose $K$ is a nonempty compact convex subset of $R^{n} ; T: K \rightarrow 2^{R^{m}}$ is a continuous, convex, and compact-valued set-valued mapping. Assume that the following conditions hold:

(i) $z \mapsto \psi(z, \cdot)$ is lower-hemicontinuous;

(ii) $\phi$ is a continuous function. 
If the solution of the BVI (1)-(2) exists, then the sequence $\left\{\left(x_{k}, x_{k}\right)\right\}$ generated by the proposed Algorithm 1 converges to a solution of the BVI (1)-(2).

Proof According to Algorithm 1 we have

$$
g(n, m) \leq \frac{1}{\mu_{k}} g\left(z_{k}, x_{k}\right) .
$$

This shows that

$$
g\left(z_{k+1}, x_{k+1}\right) \leq \prod_{j=0}^{k} \frac{1}{\mu_{j}} g\left(z_{0}, x_{0}\right) .
$$

Observe that $\mu_{k}>1$, then $k \nearrow+\infty, \prod_{i=0}^{k} \frac{1}{\mu_{j}} \searrow 0$. Since $T: K \rightarrow 2^{R^{m}}$ is convex compactvalued, from condition (i), we know that $\alpha\left(z_{0}, x_{0}\right)$ is finite. Similarly, since $K$ is nonempty compact convex subset of $R^{n}$, from condition (ii), we see that $\beta\left(z_{0}, x_{0}\right)$ is finite. Further,

$$
g\left(z_{0}, x_{0}\right)=\beta\left(z_{0}, x_{0}\right)+\alpha\left(z_{0}, x_{0}\right)
$$

is finite. This combined with $k \nearrow+\infty$ shows that we have $\prod_{j=1}^{k} \frac{1}{\mu_{j}} g\left(z_{0}, x_{0}\right) \searrow 0$.

Observe that $\left\{x_{k}\right\}_{k \in Z^{+}} \subseteq K$ is compact. Without loss of generality, let $x_{k} \rightarrow x^{*} \in K$. Owing to $T: K \rightarrow 2^{R^{m}}$ being convex compact-valued and continuous, there exists $z^{*} \in T\left(x^{*}\right)$ such that $z_{k} \rightarrow z^{*}$ and $\lim _{k \rightarrow \infty} T\left(x_{k}\right)=T\left(x^{*}\right)$. By condition (i), we have

$$
\alpha\left(z^{*}, x^{*}\right) \leq \limsup _{k \rightarrow+\infty} \alpha\left(z_{k+1}, x_{k+1}\right)
$$

Similarly, by condition (ii), we have

$$
\beta\left(z^{*}, x^{*}\right)=\limsup _{k \rightarrow+\infty} \beta\left(z_{k+1}, x_{k+1}\right) .
$$

From (20) and (19)

$$
\begin{aligned}
g\left(z^{*}, x^{*}\right) & =\alpha\left(z^{*}, x^{*}\right)+\beta\left(z^{*}, x^{*}\right) \leq \limsup _{k \rightarrow+\infty}\left(\alpha\left(z_{k+1}, x_{k+1}\right)+\beta\left(z_{k+1}, x_{k+1}\right)\right) \\
& =\limsup _{k \rightarrow+\infty} g\left(z_{k+1}, x_{k+1}\right) .
\end{aligned}
$$

By equality (18), we have

$$
\limsup _{k \rightarrow+\infty} g\left(z_{k+1}, x_{k+1}\right) \leq \limsup _{k \rightarrow+\infty} \prod_{i=0}^{k} \frac{1}{\mu_{j}} g\left(z_{0}, x_{0}\right)=0,
$$

and combining inequality (21) and (22) it is easy to see that

$$
g\left(z^{*}, x^{*}\right)=\beta\left(z^{*}, x^{*}\right)+\alpha\left(z^{*}, x^{*}\right) \leq 0,
$$

that is, $\left(z^{*}, x^{*}\right) \in S$. According to Theorem 4.1, it follows that $\left(z^{*}, x^{*}\right) \in \Theta$. So, the sequence $\left\{\left(z_{k}, x_{k}\right)\right\}$ generated by Algorithm 1 converges to a solution of the BVI. 
In order to solve the bilevel variational inequalities which has more than one solution, we modify Algorithm 1 to obtain Algorithm 2.

\section{Algorithm 2}

Step 1. Take $\left\{\mu_{k}^{j}\right\}_{k \in Z^{+}} \subset(1,+\infty), j \in Z^{+}$, where $Z^{+}$is the set of all nonnegative integers. Let the tolerance $\varepsilon \geq 0, k=0, j=1$. Choose $x_{0}^{1} \in K$ arbitrarily, compute $z_{0}^{1}=$ $\arg \min _{z \in T\left(x_{0}^{1}\right)} g\left(x_{0}^{1}, z\right)$, such that $g\left(z_{0}^{1}, x_{0}^{1}\right)>\varepsilon$, let $K=K \backslash x_{0}^{1}$. Go to Step 2 .

Step 2. Take $m \in K$, let $K=K \backslash m$. Compute $n=\arg \min _{z \in T(m)} g(z, m)$, go to Step 3 .

Step 3. If

$$
g(n, m) \leq \frac{1}{\mu_{k}^{j}} g\left(z_{k}^{j}, x_{k}^{j}\right)
$$

let $z_{k+1}^{j}=n, x_{k+1}^{j}=m$, go to Step 4. Otherwise, go to Step 2 .

Step 4. If

$$
g\left(z_{k+1}^{j}, x_{k+1}^{j}\right) \leq \varepsilon
$$

put out $\left(z_{k+1}^{j}, x_{k+1}^{j}\right), j=j+1$, choose $x_{0}^{j} \in K$, compute $z_{0}^{j}=\arg \min _{z \in T\left(x_{0}^{j}\right)} g\left(x_{0}^{j}, z\right)$ such that $g\left(z_{0}^{j}, x_{0}^{j}\right)>\varepsilon$ (automatically initialize $x_{0}^{j}$ and $z_{0}^{j}$ again), take $\left\{\mu_{k}^{j}\right\}_{k \in Z^{+}} \subset(1,+\infty)$, let $K=K \backslash x_{0}^{j}$, go to Step 5. Otherwise, let $k=k+1$, go to Step 5 .

Step 5. If $K=\emptyset$, stop. Otherwise, go to Step 2 .

Remark 5.3 In Step 2 we discretize $K$ within an acceptable error range of solution. $m$ takes different discrete points $\mathrm{m}$ of $K$ in every iterative process. It is not hard to see that the major part of Algorithm 2 is similar to Algorithm 1. If we use Algorithm 1 to solve the BVI which has more than one solution, Algorithm 1 will stop when we get one solution. In order to get all the solutions, we should choose another initial point again, and reiterate the process of Algorithm 1. So, Step 4 in Algorithm 2 is important. $m$ needs to take all the discrete points of $K$. Since we discretize $K$ within an acceptable error range of solution, the stopping criterion $K=\emptyset$ is reasonable.

Theorem 5.2 Suppose all the conditions of Theorem 5.1 are satisfied. If the BVI (1)-(2) has $n$ solutions, then, for all $j \in[1, n]$ the sequence $\left\{\left(x_{k}^{j}, x_{k}^{j}\right)\right\}$ generated by the proposed Algorithm 2 converges to one solution of the BVI (1)-(2). Further, Algorithm 2 can be used to find all the solution of the BVI (1)-(2).

Proof The proof of Theorem 5.1 is similar to the proof of Theorem 5.2, so it is omitted here.

\section{Numerical illustrative examples}

Next, we will given two numerical examples of the proposed Algorithm 1.

Example 6.1 Let $R=(-\infty,+\infty), K=[1,2]$ and $T(x)=[1, x]$ for $x \in K, z, v, y \in R$, $\langle H(z, x), x-y\rangle=\langle z x, x-y\rangle=z x(x-y),\langle P(z), z-v\rangle=\langle z, z-v\rangle=z(z-v)$. The BVI is defined as follows: find $x \in K$ such that

$$
z x(x-y) \leq 0, \quad \forall y \in K
$$


where $z$ is the solution of the following variational inequality: find $z \in T(x)=[1, x]$ such that

$$
z(z-v) \leq 0, \quad \forall v \in T(x)
$$

It is easy to see that the lower-level solution set is $M(x)=\{1\}, \forall x \in K$, and $(1,1)$ is the unique solution of the BVI (23)-(24).

The gap function of the lower level variational inequality is

$$
\alpha(z, x)=\sup _{v \in[1, x]} z(z-v)=z(z-1) .
$$

The gap function of the upper-level variational inequality is

$$
\beta(z, x)=\sup _{y \in[1,2]} x z(x-y)=x z(x-1) .
$$

Further,

$$
g(z, x)=\beta(z, x)+\alpha(z, x)=x z(x-1)+z(z-1) .
$$

Example 6.2 Let $R=(-\infty,+\infty), K=[0.5,5]$, and $T(x)=[0.5,2 x]$ for $x \in K, z, v, y \in R$, $\langle H(z, x), x-y\rangle=\left\langle-3 z x^{2}, x-y\right\rangle=-3 z x^{2}(x-y),\langle P(z), z-v\rangle=\langle 3 z, z-v\rangle=3 z(z-v)$. The BVI is defined as follows: find $x \in K$ such that

$$
-3 z x^{2}(x-y) \leq 0, \quad \forall y \in K,
$$

where $z$ is the solution of the following variational inequality: find $z \in T(x)=[0.5,2 x]$ such that

$$
3 z(z-v) \leq 0, \quad \forall v \in T(x) .
$$

It is easy to see that the rational reaction set is $M(x)=\{0.5\}, \forall x \in K$, and $(0.5,5)$ is the unique solution of the BVI (25)-(26).

The gap function of the lower level variational inequality is

$$
\alpha(z, x)=\sup _{v \in[0.5,2 x]} 3 z(z-v)=3 z(z-0.5) .
$$

The gap function of the upper-level variational inequality is

$$
\beta(z, x)=\sup _{y \in[0.5,5]}-3 x z^{2}(x-y)=-3 x z^{2}(x-5) .
$$

Further,

$$
g(z, x)=\beta(z, x)+\alpha(z, x)=-3 x z^{2}(x-5)+3 z(z-0.5) .
$$


Table 1 Computational results using the proposed Algorithm 1

\begin{tabular}{lllllll}
\hline Example & $\boldsymbol{x}_{\mathbf{0}}$ & $\boldsymbol{\mu}$ & $\boldsymbol{\varepsilon}$ & $(\mathbf{z}, \boldsymbol{x})$ & $\boldsymbol{g ( z ,}, \boldsymbol{x})$ & times \\
\hline 6.1 & 1.3 & 1.1 & 0.001 & $(1.000,1.000)$ & 0.0001 & $0.714 \mathrm{~s}$ \\
6.2 & 3.5 & 1.1 & 0.001 & $(0.500,5.007)$ & 0.0006 & $0.752 \mathrm{~s}$ \\
\hline
\end{tabular}

Using the proposed Algorithm 1, we obtain the results for solving Example 6.1 and Example 6.2. In Table 1, the fifth column denotes the solutions of the BVI (23)-(24) and the BVI (25)-(26); the sixth column denotes the value of $g(z, x)$, which corresponds to equality to the BVI; the third column denotes the value of $\mu_{k}$, here we let $\mu_{k}$ be a fixed value $\mu$; the last column denotes the operation time. From the numerical results, we observe that the proposed algorithm can solve the BVI well.

Next, we will given two numerical examples for the proposed Algorithm 2.

Example 6.3 Let $R=(-\infty,+\infty), K=[1,2]$, and $T(x)=[1,2+x]$ for $x \in K, z, v, y \in R$, $\langle H(z, x), x-y\rangle=\langle z(z-1.2) x, x-y\rangle=z(z-1.2) x(x-y),\langle P(z), z-v\rangle=\left\langle z(z-1.5)^{2}, z-v\right\rangle=$ $z(z-1.5)^{2}(z-v)$. The BVI is defined as follows: find $x \in K$ such that

$$
z(z-1.2) x(x-y) \leq 0, \quad \forall y \in K,
$$

where $z$ is the solution of the following variational inequality: find $z \in T(x)=[1,2+x]$ such that

$$
z(z-1.5)^{2}(z-v) \leq 0, \quad \forall v \in T(x) .
$$

It is easy to see that the lower-level solution set is $M(x)=\{1,1.5\}, \forall x \in K . M(x)$ is not a singleton, and $\{(1,2),(1.5,1)\}$ is the solution set of the BVI (27)-(28).

The gap function of the lower level variational inequality is

$$
\alpha(z, x)=\sup _{v \in[1,2+x]} z(z-1.5)^{2}(z-v)=z(z-1.5)^{2}(z-1) .
$$

The gap function of the upper-level variational inequality is

$$
\beta(z, x)=\sup _{y \in[1,2]} z(z-1.2) x(x-y) .
$$

Moreover,

$$
\sup _{y \in[1,2]} z(z-1.2) x(x-y)= \begin{cases}x z(z-1.2)(x-2), & z \leq 1.2 \\ x z(z-1.2)(x-1), & z>1.2 .\end{cases}
$$

Further,

$$
g(z, x)=\beta(z, x)+\alpha(z, x)= \begin{cases}x z(z-1.2)(x-2)+z(z-1.5)^{2}(z-1), & z \leq 1.2 \\ x z(z-1.2)(x-1)+z(z-1.5)^{2}(z-1,), & z>1.2\end{cases}
$$


Example 6.4 Let $R=(-\infty,+\infty), K=[1,4]$, and $T(x)=[1,4+2 x]$ for $x \in K, z, v, y \in R$, $\langle H(z, x), x-y\rangle=\langle(z-1.5) x, x-y\rangle=(z-1.5) x(x-y),\langle P(z), z-v\rangle=\left\langle z^{2}(z-2)^{2}, z-v\right\rangle=z^{2}(z-$ $2)^{2}(z-v)$. The BVI is defined as follows: find $x \in K$ such that

$$
(z-1.5) x(x-y) \leq 0, \quad \forall y \in K,
$$

where $z$ is the solution of the following variational inequality: find $z \in T(x)=[1,4+2 x]$ such that

$$
z^{2}(z-2)^{2}(z-v) \leq 0, \quad \forall v \in T(x)
$$

It is easy to see that the lower-level solution set is $M(x)=\{1,2\}, \forall x \in K . M(x)$ is not a singleton, and $\{(1,4),(2,1)\}$ is the solution set of the BVI (29)-(30).

The gap function of the lower level variational inequality is

$$
\alpha(z, x)=\sup _{v \in[1,4+2 x]} z^{2}(z-2)^{2}(z-v)=z^{2}(z-2)^{2}(z-1) .
$$

The gap function of the upper-level variational inequality is

$$
\beta(z, x)=\sup _{y \in[1,4]}(z-1.5) x(x-y) .
$$

Moreover,

$$
\sup _{y \in[1,4]}(z-1.5) x(x-y)= \begin{cases}(z-1.5) x(x-4), & z \leq 1.5, \\ (z-1.5) x(x-1), & z>1.5 .\end{cases}
$$

Further,

$$
g(z, x)=\beta(z, x)+\alpha(z, x)= \begin{cases}(z-1.5) x(x-4)+z^{2}(z-2)^{2}(z-1), & z \leq 1.5 \\ (z-1.5) x(x-1)+z^{2}(z-2)^{2}(z-1), & z>1.5 .\end{cases}
$$

Example 6.5 Let $R=(-\infty,+\infty), K=[1,2] \times[1,2]$, and $T(x)=\left[1,2+x_{1}\right]$ for $x \in K, z, v, y \in$ $R,\langle H(z, x), x-y\rangle=\left((1.2-z) x_{1}, 8\right)\left(x_{1}-y_{2}, x_{2}-y_{2}\right)^{\top},\langle P(z), z-v\rangle=(z-1.4)^{2}(z-v)$. The BVI is defined as follows: find $x \in K$ such that

$$
\left((1.2-z) x_{1}, 8\right)\left(x_{1}-y_{2}, x_{2}-y_{2}\right)^{\top} \leq 0, \quad \forall y \in K,
$$

where $z$ is the solution of the following variational inequality: find $z \in T(x)=\left[1,2+x_{1}\right]$ such that

$$
(z-1.4)^{2}(z-v) \leq 0, \quad \forall v \in T(x)
$$

It is easy to see that the lower-level solution set is $M(x)=\{1,1.4\}, \forall x \in K . M(x)$ is not a singleton, and $\{((2,1), 1.4),((1,1), 1)\}$ is the solution set of the BVI $(29)-(30)$. 
Table 2 Computational results using the proposed Algorithm 2

\begin{tabular}{llllll}
\hline Example & $\boldsymbol{x}_{\mathbf{0}}^{\boldsymbol{j}}$ & $\boldsymbol{\mu}^{\boldsymbol{j}}$ & $(\mathbf{z}, \boldsymbol{x})$ & $\boldsymbol{g ( z , \boldsymbol { x } )}$ & times \\
\hline 6.3 & 1.9 & 1.1 & $(1.498,1.002)$ & 0.0008 & $2.674 \mathrm{~s}$ \\
& 1.91 & 1.1 & $(1.000,1.998)$ & 0.0009 & \\
6.4 & 1.6 & 1.1 & $(1.997,1.002)$ & 0.0010 & $7.618 \mathrm{~s}$ \\
& 1.61 & 1.1 & $(1.000,4.000)$ & 0.0000 & \\
6.5 & $(2,2)$ & 1.1 & $(1.402,(2,1.001))$ & 0.008 & $75.260 \mathrm{~s}$ \\
& $(2,0.99)$ & 1.1 & $(1.005,(1,1.001))$ & 0.008 & \\
\hline
\end{tabular}

Table 3 Compare Algorithm 1 with Algorithm 2 when solve Example 6.1, respectively

\begin{tabular}{lllllll}
\hline method & $\mathbf{x}_{\mathbf{0}}$ & $\boldsymbol{\mu}$ & $\boldsymbol{\varepsilon}$ & $(\mathbf{z}, \boldsymbol{x})$ & $\boldsymbol{g ( z , \boldsymbol { x } )}$ & times \\
\hline Algorithm 1 & 1.3 & 1.1 & 0.001 & $(1.000,1.000)$ & 0.0001 & $0.714 \mathrm{~s}$ \\
Algorithm 2 & 1.3 & 1.1 & 0.001 & $(1.000,1.000)$ & 0.0001 & $2.556 \mathrm{~s}$
\end{tabular}

The gap function of the lower level variational inequality is

$$
\alpha(z, x)=\sup _{v \in\left[1,2+x_{1}\right]}(z-1.4)^{2}(z-v)=(z-1.4)^{2}(z-1) .
$$

The gap function of the upper-level variational inequality is

$$
\begin{aligned}
\beta(z, x) & =\sup _{y \in[1,2] \times[1,2]}\left((1.2-z) x_{1}, 8\right)\left(x_{1}-y_{2}, x_{2}-y_{2}\right)^{\top} \\
& =(1.2-z) x_{1}\left(x_{1}-y_{1}\right)+8\left(x_{2}-y_{2}\right) .
\end{aligned}
$$

Moreover,

$$
\sup _{y \in[1,2] \times[1,2]}(1.2-z) x_{1}\left(x_{1}-y_{1}\right)+8\left(x_{2}-y_{2}\right)= \begin{cases}(1.2-z) x_{1}\left(x_{1}-1\right)+8\left(x_{2}-1\right), & z \leq 1.2, \\ (1.2-z) x_{1}\left(x_{1}-2\right)+8\left(x_{2}-1\right), & z>1.2 .\end{cases}
$$

Let $\varepsilon=0.01$, use the proposed Algorithm 2, we obtain the results for solving Example 6.3, Example 6.4, and Example 6.5. In Table 2, the fourth column denotes the solutions of the BVI (27)-(28), the BVI (29)-(30), and the BVI (31)-(32); the fifth column denotes the value of $g(z, x)$ which is equivalent to the BVI; the second column denotes the initial value of $x_{0}^{j}$. From this column we can see that, in order to get the approximate solution of Example 6.3, Algorithm 2 only needs to initialize $x_{0}$ two times, that is, $x_{0}^{1}=1.9, x_{0}^{2}=1.91$; the third column denotes the value of $\mu_{k}^{j}$, here we let $\mu_{k}^{j}=\mu^{j}$ be a fixed value 1.1; the last column denotes the operation time. From the numerical results, we observe that the proposed Algorithm 2 can get all solutions of the BVI.

Next, we solve the BVI (23)-(24) which has only one solution in Example 6.1 with the two proposed algorithms, respectively; see the results detailed in Table 3.

In Table 3, we can see that the two algorithms can solve the BVI (23)-(24) well. The last column denotes the operation time. Obviously, Algorithm 1 is faster than Algorithm 2. Because the two algorithms have different termination conditions, if the BVI has only one solution, we should solve it by Algorithm 1. 


\section{Conclusions}

In this paper, we have investigated a class of bilevel variational inequalities (BVIs) with hierarchical nesting structure, which was introduced in [25] first. In the case that the solution set $M(x)$ of the lower-level variational inequality is not a singleton, we call it Illposed bilevel variational inequality. We first obtained the existence theorem of a solution for ill-posed bilevel variational inequalities by the Himmelberg fixed point theorem. Then the uniqueness of a solution for the upper-level variational inequality in regard to every feedback parameter is given under some mild condition. We transform the BVI into a onelevel variational inequality by gap functions of the upper-level and lower-level function, and we proved their equivalence. Two algorithms to find the solutions of the BVI were constructed. Finally, we have proved the convergence of the iterative sequence generated by the proposed algorithm under some mild conditions. We said that the two algorithms can also be used to solve well-posed bilevel variational inequalities. Furthermore, we can see that for the two algorithms $M(x)$ does not need to be compact or convex. In the future, we will consider the following questions. We know that one of the conditions for a solution to exist is that $M(x)$ is a convex set. In the future we will consider the solution existence conditions in the case that $M(x)$ is not a convex set. Since the BVI is a very complicated model, it is quite difficulty to design an algorithm that is efficient for all examples. From the numerical examples, we can see that the two algorithms are efficient in $R$. In the future, we will design algorithms that are more efficient in $R^{d}$ for $d>2$.

Competing interests

The authors declare that they have no competing interests.

Authors' contributions

All authors contributed equally to the writing of this paper. All authors read and approved the final manuscript.

\section{Author details}

${ }^{1}$ School of Mathematics and Statistics, Wuhan University, Wuhan, 430072, China. ${ }^{2}$ Computational Science Hubei Key Laboratory, Wuhan University, Wuhan, 430072, China. ${ }^{3}$ School of Mathematics and Statistics, Southwest University,

Chongqing, 400715, China.

\section{Acknowledgements}

This work of Z Wan was partially supported by the Natural Science Foundation of China, No. 71471140 and No. 71171150. The work of J-w Chen was partially supported by the Natural Science Foundation of China, No. 11401487. The work of G Li was partially supported by Graduate Students Independent Research Foundation of Wuhan University, No. 2015201020204.

Received: 6 October 2015 Accepted: 3 March 2016 Published online: 25 March 2016

\section{References}

1. Dempe, S: Foundations of Bilevel Programming. Kluwer Academic, Dordrecht (2002)

2. Stackelberg, HV: Marktform und gleichgewicht. Springer, Berlin (1934)

3. Andreani, R, Martı, JM, et al.: On the solution of mathematical programming problems with equilibrium constraints. Math. Methods Oper. Res. 54(3), 345-358 (2001)

4. Dempe, S, Dutta, J: Is bilevel programming a special case of a mathematical program with complementarity constraints? Math. Program. 131(1-2), 37-48 (2012)

5. Dempe, S, Zemkoho, AB: The bilevel programming problem: reformulations, constraint qualifications and optimality conditions. Math. Program. 138(1-2), 447-473 (2013)

6. Dinh, BV, Muu, LD: Algorithms for a class of bilevel programs involving pseudomonotone variational inequalities. Acta Math. Vietnam. 38(4), 529-540 (2013)

7. Ye, J, Zhu, D, Zhu, Q: Exact penalization and necessary optimality conditions for generalized bilevel programming problems. SIAM J. Optim. 7(2), 481-507 (1997)

8. Ye, JJ: Nondifferentiable multiplier rules for optimization and bilevel optimization problems. SIAM J. Optim. 15(1), 252-274 (2004)

9. Yin, H, Zhang, J: Global convergence of a smooth approximation method for mathematical programs with complementarity constraints. Math. Methods Oper. Res. 64(2), 255-269 (2006)

10. Bard, JF, Plummer, J, Claude Sourie, J: A bilevel programming approach to determining tax credits for biofuel production. Eur. J. Oper. Res. 120(1), 30-46 (2000) 
11. Lu, J, Shi, C, Zhang, G: On bilevel multi-follower decision making: general framework and solutions. Inf. Sci. 176(11), 1607-1627 (2006)

12. Chiou, S-W: Bilevel programming for the continuous transport network design problem. Transp. Res., Part B, Methodol. 39(4), 361-383 (2005)

13. Nair, R, Miller Hooks, E: Equilibrium network design of shared-vehicle systems. Eur. J. Oper. Res. 235(1), $47-61$ (2014)

14. Hobbs, BF, Nelson, SK: A nonlinear bilevel model for analysis of electric utility demand-side planning issues. Ann. Oper. Res. 34(1), 255-274 (1992)

15. Nicholls, MG: Aluminum production modeling a nonlinear bilevel programming approach. Oper. Res. 43(2), 208-218 (1995)

16. Roghanian, E, Sadjadi, SJ, Aryanezhad, M-B: A probabilistic bi-level linear multi-objective programming problem to supply chain planning. Appl. Math. Comput. 188(1), 786-800 (2007)

17. Wang, $X$, Wang, $Y, C$ Cui, $Y$ : A new multi-objective bi-level programming model for energy and locality aware multi-job scheduling in cloud computing. Future Gener. Comput. Syst. 36, 91-101 (2014)

18. Anh, PN, Kim, J, Muu, LD: An extragradient algorithm for solving bilevel pseudomonotone variational inequalities. J. Glob. Optim. 52(3), 627-639 (2012)

19. Anh, TT, Long, LB, Anh, TV: A projection method for bilevel variational inequalities. J. Inequal. Appl. 2014, 205 (2014)

20. Chen, J-W, Wan, Z, Cho, YJ: The existence of solutions and well-posedness for bilevel mixed equilibrium problems in Banach spaces. Taiwan. J. Math. 17(2), 725-748 (2013)

21. Moudafi, A: Proximal methods for a class of bilevel monotone equilibrium problems. J. Glob. Optim. 47(2), 287-292 (2010)

22. Noor, MA, Huang, Z: A iterative scheme for a system of quasi variational inequalities. J. Math. Inequal. 1(1), 31-38 (2007)

23. Peng, J, Zhu, D: A new system of generalized mixed quasi-variational inclusions with $(h, \eta)$-monotone operators J. Math. Anal. Appl. 327(1), 175-187 (2007)

24. Wan, Z, Chen, J-w, Sun, $H$, Yuan, L: A new system of generalized mixed quasivariational inclusions with relaxed cocoercive operators and applications. J. Appl. Math. 2011, 961038 (2011)

25. Wan, Z, Chen, J-w: On bilevel variational inequalities. J. Oper. Res. Soc. China 1(4), 483-510 (2013)

26. Francisco, F, Christian, K, Simone, S: Solving quasi-variational inequalities via their KKT conditions. Math. Program. 144(1-2), 369-412 (2014)

27. Christian, K: On the multiplier-penalty-approach for quasi-variational inequalities. Math. Program. 1-31 (2015)

28. Noor, MA: On general quasi-variational inequalities. J. King Saud Univ., Sci. 24(1), 81-88 (2012)

29. Aubin, J-P, Ekeland, I: Applied Nonlinear Analysis. Wiley-Interscience, New York (1984)

30. Wu, X, Shen, S: A further generalization of Yannelis-Prabhakar's continuous selection theorem and its applications, J. Math. Anal. Appl. 197(1), 61-74 (1996)

31. Ding, XP, Kim, WK, Tan, K-K: A selection theorem and its applications. Bull. Aust. Math. Soc. 46(2), 205-212 (1992)

32. Alghamdi, MA, Shahzard, N, Valero, O: On fixed point theory in partial metric spaces. Fixed Point Theory Appl. 2012, 1 (2012)

33. Hidetoshi, K, Sehie, P: Remarks on extensions of the Himmelberg fixed point theorem. Fixed Point Theory Appl. 2007, $016028(2007)$

34. Horsley, A, Wrobel, AJ, Van Zandt, T: Berge's maximum theorem with two topologies on the action set. Econ. Lett. 61(3), 285-291 (1998)

\section{Submit your manuscript to a SpringerOpen ${ }^{\circ}$ journal and benefit from:}

- Convenient online submission

Rigorous peer review

- Immediate publication on acceptance

- Open access: articles freely available online

- High visibility within the field

- Retaining the copyright to your article 